\title{
Reef fish community in presence of the lionfish (Pterois volitans) in Santa Marta, Colombian Caribbean
}

\section{Comunidad de peces arrecifales en presencia del pez león (Pterois volitans) en Santa Marta, Caribe colombiano}

\author{
Rocío García-Urueña, ${ }^{1 *}$ Ph.D, Arturo Acero P, ${ }^{2}$ Ph.D, Víctor Coronado-Carrascal, ${ }^{1}$ B.Sc.
}

\begin{abstract}
${ }^{1}$ Universidad del Magdalena, Grupo de Investigación Ecología y Diversidad de Algas Marinas y Arrecifes Coralinos. Carrera 32 \# 22-08, Santa Marta, Colombia. ${ }^{2}$ Instituto de Estudios en Ciencias del Mar (CECIMAR). Universidad Nacional de Colombia sede Caribe, El Rodadero, Santa Marta, Colombia. *Correspondencia: garciarociop@yahoo.com
\end{abstract}

Received: October 2014; Acepted: March 2015.

\begin{abstract}
Objective. Fish species community structure and benthic organisms coverage were studied in five localities in Santa Marta where the lionfish is present. Materials and methods. Abundance of fish species, including lion fish, was established using 30 m random visual censuses and video transects; trophic guilds were established according to available references. On the other hand benthic coverage was evaluated using the software Coral Point Count (CPCe) 4.0. Results. Families with higher species

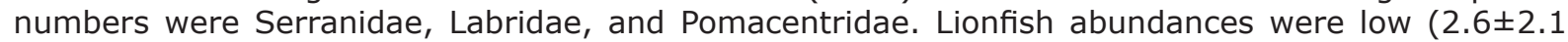
ind/120 $\mathrm{m}^{2}$ ), but in any case Pterois volitans was observed as the eleventh more abundant species, surpassing species of commercial value such as Cephalopholis cruentata. Species that were found in larger numbers ( $>100$, Thalassoma bifasciatum, Haemulon aurolineatum, Canthigaster rostrata, Abudefduf saxatilis, Chromis cyanea, and Stegastes partitus) were mainly invertebrate eaters, planctivores, and territorial herbivores. Coral coverage showed higher coral percentages in Chengue (69.9\%) and Cinto (27.4\%), larger sponge percentages in Morro (32.7\%); Isla Aguja and Remanso showed the larger figures for abiotic substrate ( 41.6 and $37 \%$, respectively); corals, sponges, and gorgonians were the components best explaining fish community, but not for the lion fish, which inhabit all studied reef formations. Conclusions. Lion fish is ranked between the 20 more abundant species, with none commercially important species larger, hence no species may qualify as a natural control. Lion fish has as well become a relatively abundant species in Santa Marta reefs, independent of benthic coverage.
\end{abstract}

Key words: Exotic species, marine ecology, marine fishes (Source: IEDCYT).

\section{RESUMEN}

Objetivo. Se estudió la estructura de especies de la comunidad íctica y se evaluó la cobertura de organismos bentónicos en cinco localidades en Santa Marta, Colombia con presencia del pez león. Materiales y métodos. Se estimó la abundancia a través de censos visuales y video transectos de $30 \mathrm{~m}$ y se estableció el gremio trófico de cada especie según literatura. El sustrato se cuantificó con el software Coral Point Count (CPCe) 4.0. Resultados. Las familias con más especies fueron Serranidae, Labridae y Pomacentridae $y$, aunque su abundancia promedio fue baja $\left(2.6 \pm 2.1 \mathrm{ind} / 120 \mathrm{~m}^{2}\right)$, Pterois 
volitans fue la undécima especie más abundante, superando a peces comerciales como Cephalopholis cruentata. Las especies más abundantes fueron invertívoros, planctívoros y herbívoros, tales como Thalassoma bifasciatum, Haemulon aurolineatum, Canthigaster rostrata, Abudefduf saxatilis, Chromis cyanea y Stegastes partitus. Se encontró mayor porcentaje de coral en Chengue (69.9\%) y Cinto (27.4\%) y de esponjas para Morro (32.7\%); para Isla Aguja y Remanso predominó el componente abiótico (41.6 y $37 \%$, respectivamente). Corales, esponjas y gorgonias fueron los componentes que mejor explicaron la comunidad íctica, aunque no para el pez león que habita todas las formaciones arrecifales. Conclusiones. El pez león se ubica entre las 20 especies de peces más abundante, con ninguna especie de importancia comercial en los sitios muestreados que lo supere en talla y que pudiera ser un control natural del invasor. Igualmente, se ha convertido en una especie relativamente abundante en los arrecifes samarios sin importar los atributos del fondo.

Palabras clave: Ecología marina, especies exóticas, peces marinos (Fuente: IEDCYT).

\section{INTRODUCTION}

Since the mid-1970s, Acero y Garzón (1) have gathered information on the biodiversity of marine fish in the Santa Marta area, recording 372 species. Later, Acero (2) listed commercial species present in the Caribbean region of continental Colombia, citing a total of 449 species. These records are greatly relevant since they show the high number of species from the Lutjanidae, Serranidae, Carangidae, Haemulidae and Scaridae families.

To evaluate the deterioration of populations, Grijalba-Bendeck et al (3) analyzed different fishing techniques and disembarking points to determine priority species and conservation areas. Lutjanus analis and Mycteroperca interstialis were catalogued as vulnerable, and Epinephelus striatus as in danger of extinction. The most important factors that led to this condition were overfishing, deterioration, pollution, anthropogenic habitat alterations, and fishing below the minimum size for sexual maturity. In 2013 Álvarez et al (4) mentioned that fishing with increasingly more effective and less selective methods in the reefs of the Caribbean region of Colombia have meant that carnivorous fish have reached very critical levels where species such as Serranidae (groupers Epinephelus spp., Mycteroperca spp. and Cephalopholis spp.), Lutjanidae (snappers Lujtanus spp.) and Centropomidae (snooks Centropomus spp.) have practically disappeared or have been greatly reduced.

This problem has worsened with the recent lionfish (Pterois volitans) invasion. This species, native to the Indo-Pacific region, has become established with remarkable success in the western Atlantic Ocean, including the Caribbean Sea $(5,6)$. Presently, a greater biomass and density has been recorded in the invaded area than with the nine lionfish (Pterois volitans) species occurring in their original distribution

\section{INTRODUCCIÓN}

Desde mediados de la década de los años 70 del siglo pasado, Acero y Garzón (1) reunieron información sobre la biodiversidad de peces marinos para el área de Santa Marta registrando 372 especies. Luego Acero (2) lista las especies comerciales del Caribe continental colombiano citando un total de 449 especies. Estos registros son de gran relevancia debido a que en ellos se puede ver el alto número de especies de las familias Lutjanidae, Serranidae, Carangidae, Haemulidae y Scaridae conocidas.

Para evaluar el deterioro de las poblaciones, Grijalba-Bendeck et al (3) analizaron diferentes artes de pesca y sitios de desembarque, determinando especies y áreas prioritarias para la conservación. Lutjanus analis y Mycteroperca interstialis fueron especies catalogadas como vulnerables y Epinephelus striatus en peligro de extinción, siendo la presión pesquera, degradación, contaminación, la alteración antropogénica de los hábitats y la captura por debajo de talla mínima de madurez sexual los factores más importantes del estado actual.

Para 2013, Álvarez et al (4) mencionan que la pesca con métodos cada día más efectivos y por lo tanto menos selectivos en los arrecifes del Caribe colombiano, ha llevado a los peces carnívoros a niveles muy críticos, donde especies como serránidos (meros-Epinephelus spp., chernas-Mycteroperca spp., mamitasCephalopholis spp.), lutjánidos (pargos-Lujtanus spp.) y centropómidos (róbalos-Centropomus spp.) han prácticamente desaparecido o están enormemente reducidos.

A la problemática actual se suma la reciente invasión biológica del pez león (Pterois volitans). Esta especie nativa del Indo-Pacifico logró establecerse con gran éxito en el Atlántico occidental y el mar Caribe $(5,6)$. Actualmente, se ha registrado una densidad y biomasa mayor 
area $(7,8)$. Apparently, this fish has devastating effects on native communities and could be one of the most harmful marine invasions that has ever been recorded (9). It is considered that in as little as five weeks $P$. volitans is capable of reducing the recruitment of native fish in small areas (5) by $80 \%$, possibly affecting species that fulfill important ecological roles, such as herbivores (e.g Labridae and Pomacentridae). Similarly, this species could compete for food and space with other species that are economically important, such as snappers (Lutjanidae) and groupers (Serranidae), diminishing quantities and recruitment $(5,7,10)$. Presently, the lionfish is considered worldwide as one of the 10 species that has the greatest ecological and economic impact on invaded sites (11).

In the Caribbean region of continental Colombia, González et al (12) were the first to document the presence of lionfish in the Tayrona National Park (Parque Nacional Natural Tayrona, PNNT), and since then the numbers of this fish have increased dramatically. In Colombia, it is considered that the invasion could contribute even further to reducing fish populations in the region, and even more so because a large number of variables make the problem even more complex $(3,4)$. The objective of this study was to evaluate the composition in the fishing community and the availability of substrate both for the invading species as well as the native reef species in order to provide information that can be used as a reference for possible changes in the structure of the fish community, if there are any.

\section{MATERIALS AND METHODS}

Area of study. Five reef formations were studied: one located in the Santa Marta bay (Morro), another at the limit of Taganga Bay and Tayrona National Park (TNP) (Remanso), and three in the TNP (Isla Aguja, Chengue, and Cinto). Morro is at $11^{\circ} 14^{\prime} \mathrm{N}$ and $74{ }^{\circ} 14^{\prime} \mathrm{W}$, and its waters are constantly polluted from several sources: Boquerón (sewage water from the city), seaport (port and industry wastes), and Manzanares River (sediment and domestic waste), so that the reef patches only cover 20 to $28 \%$ (13).

Remanso, located at $11^{\circ} 16^{\prime} \mathrm{N}$ and $74^{\circ} 12^{\prime} \mathrm{W}$, is at the extreme northeast of Taganga, with a primarily rocky shore and a shallow bordering reef (14). Geological formations on Isla Aguja $\left(11^{\circ} 19^{\prime} \mathrm{N}, 7^{\circ} 12^{\prime} \mathrm{W}\right)$ are rocky-coral substrate, with an average depth of $25 \mathrm{~m}$ and a reef that is slightly inclined, of compact rock and stones (14). Chengue is a bay that is located in TNP at en el área invadida que la de las nueve especies registradas de pez león (Pterois $s p p$ ) en su área de distribución $(7,8)$. Al parecer este pez tiene fuertes efectos sobre las comunidades nativas y puede ser una de las invasiones marinas más nocivas de la que se tenga registro (9). Se considera que $P$. volitans en un tiempo tan corto como cinco semanas es capaz de reducir el reclutamiento de peces nativos en pequeños parches cerca de un $80 \%$ (5), pudiendo afectar especies que cumplen funciones ecológicas importantes como la herbivoría (e.g Labridae y Pomacentridae). Asimismo, esta especie podría competir por alimento y espacio, con otras especies de importancia económica como pargos (Lutjanidae) y meros (Serranidae), disminuyendo su abundancia y reclutamiento $(5,7,10)$. En la actualidad, el pez león es considerado como una de las 10 especies a nivel mundial con mayor impacto ecológico y económico en los sitios invadidos (11).

En el Caribe continental colombiano la presencia del pez león fue documentada por primera vez por González et al (12) en el Parque Nacional Natural Tayrona (PNNT) y actualmente su abundancia ha aumentado dramáticamente. En Colombia se considera que la invasión podría contribuir aún más a la disminución de las poblaciones de peces en la región, más aun como se ha mencionado, existe un gran número de variables que hacen la problemática aún más compleja $(3,4)$. El objetivo de estudio fue evaluar la composición de la comunidad íctica y la disponibilidad de sustrato tanto para la especie invasora como para las nativas de peces arrecifales, con el propósito de aportar información que permita ser un referente de posibles cambios en la estructura de la comunidad íctica, si es que los hay.

\section{MATERIALES Y MÉTODOS}

Área de estudio. Cinco formaciones arrecifales fueron estudiadas; una correspondiente a la bahía de Santa Marta (Morro), otra al límite entre la bahía de Taganga y el PNNT (Remanso) $y$ tres en el PNNT (Isla Aguja, Chengue y Cinto). Morro se localiza a $11^{\circ} 14^{\prime} \mathrm{N}$ y $74^{\circ} 14^{\prime} \mathrm{O}$ y sus aguas constantemente están influenciadas por fuentes de contaminación provenientes de varios focos como Boquerón (aguas servidas de la ciudad), Terminal Marítimo (desechos portuarios e industriales) y río Manzanares (sedimentos y residuos domésticos), de allí que los parches arrecifales solo presentan una cobertura entre 20 y $28 \%$ (13).

Remanso, localizada a $11^{\circ} 16^{\prime}>\mathrm{N}$ y $74^{\circ} 12^{\prime}>\mathrm{O}$, es un área que se encuentra en el extremo nororiental de Taganga, caracterizada por 
$11^{\circ} 19^{\prime} \mathrm{N}$ and $74^{\circ} 08^{\prime} \mathrm{W}, 14 \mathrm{~km}$ northeast of Santa Marta. The topography is heterogeneous, with a slight slope where patchy reefs are formed at a certain distance from the shore (13). The bay of Cinto is found at $11^{\circ} 20^{\prime} \mathrm{N}$ and $74^{\circ} 03^{\prime} \mathrm{W}$, and is the central part of the marine sector of TNP. Exposure to waves in this area is similar to Chengue, and both bays have predominantly clear water.

Field methods. In each locality, a permanent transect $30 \mathrm{~m}$ long and $4 \mathrm{~m}$ wide $\left(120 \mathrm{~m}^{2}\right)$ and between 10 and $12 \mathrm{~m}$ deep was marked off; between January and December 2012 the number of lionfish was recorded monthly. The fish population was recorded three times during the study period. Using scuba diving equipment, two observers performed visual census for 30 minutes, counting individual fish from all the species found. Relative quantity analysis was considered as the relationship between the number of individuals of a species and the total of all species; in the same way, the relative number of families was determined by comparing its dominance in each locality. For each species, a trophic group was established through bibliographic revisions (carnivore, invertivore, planktivore, and herbivore), (15-17).

Benthic coverage of each transect was evaluated by video in each locality, and later 30 images were frozen and assigned 50 numbers at random. The substrate was classified according to the following components (coral, gorgonian, sponges, macroalgae, dead coral, hard substrate, and unidentified). To perform this analysis the software Coral Point Count (CPCe) 4.0. was used. Finally, the species richness and Shannon-Wiever diversity indexes $\left(\mathrm{H}^{\prime}\right)$ and equitability $(\mathrm{J})$ were calculated for each locality.

Data analysis. The distribution pattern of fish species was determined using the Bray-Curtis classification index (data transformed to square root) and multidimensional scaling using the abundance matrix for each locality. To establish possible associations between the composition of substrate and abundance in the fish community, exploratory analysis of substrate components was performed and the maximum correlation was determined (Bioenv). Later, a DistLM was performed (lineal model based on distance) that included those variables that presented the highest coefficients; this test helps discover models among the fish community using environmental variables of substrate components that describe significant correlations. The analysis was done using the Primer 6 statistics program, version 6.1.13. presentar primordialmente un litoral rocoso; posee un arrecife franjeante de poca profundidad (14). Las formaciones geológicas de Isla Aguja $\left(11^{\circ} 19^{\prime}>\mathrm{N}, 74^{\circ} 12^{\circ} \mathrm{O}\right)$ son de sustrato rocosocoralino, con una profundidad promedio de 25 $\mathrm{m}$ y con un arrecife medianamente inclinado formado por rocas compactas y cantos rodados (14). Chengue es una bahía situada en el PNNT a $11^{\circ} 19>\mathrm{N}$ y $74^{\circ} 08>0$, a $14 \mathrm{~km}$ al nororiente de Santa Marta. La topografía es heterogénea, con un relieve poco escarpado donde se desarrollan arrecifes de parche a cierta distancia del litoral (13). La bahía de Cinto se encuentra ubicada a $11^{\circ} 20^{\prime} \mathrm{N}$ y $74^{\circ} 03^{\prime} \mathrm{O}$ y ocupa la parte central del sector marino del PNNT. La exposición al oleaje dentro de este sector es similar a la de Chengue, y ambas bahías se caracterizan por presentar aguas predominantemente claras.

Métodos de campo. En cada localidad, entre 10 y $12 \mathrm{~m}$ de profundidad se delimitó un transecto permanente de $30 \mathrm{~m}$ de largo por $4 \mathrm{~m}$ de ancho $\left(120 \mathrm{~m}^{2}\right)$ y mensualmente entre enero y diciembre de 2012 se registró el número de individuos de pez león. La comunidad íctica fue registrada tres veces durante el periodo de estudio. Mediante el uso de equipo de buceo autónomo dos observadores realizaron censos visuales por 30 minutos contando individuos de todas las especies de peces encontradas. Para el análisis de la abundancia relativa se consideró la relación entre el número de individuos de una especie y el total de todas las especies; igualmente se determinó la abundancia relativa de las familias para comparar la dominancia en cada una de las localidades. Para cada especie se estableció su grupo trófico a través de revisiones bibliográficas (carnívoro, invertívoro, planctívoro y herbívoro), (15-17).

La cobertura bentónica se evaluó por medio de video de cada transecto en cada localidad, para posteriormente congelar 30 imágenes y asignar 50 números aleatorios. El sustrato fue clasificado de acuerdo con los siguientes componentes (corales, gorgonias, esponjas, macroalgas, coral muerto, sustrato duro y no identificado). Para este análisis se utilizó el software Coral Point Count (CPCe) 4.0. Finalmente, se calculó la riqueza de especies y los índices de diversidad de Shannon-Wiever $\left(H^{\prime}\right)$ y equitabilidad $(J)$ para cada localidad.

Análisis de datos. El patrón de distribución de las especies de peces se determinó a través del índice de clasificación de Bray-Curtis (datos transformados a raíz cuadrada) y por escalamiento multidimensional a partir de la matriz de abundancia por localidad. Para establecer las posibles asociaciones entre la composición de 


\section{RESULTS}

A total of 88 fish species corresponding to 32 families were identified (Table 1). Of these, those that presented the greatest number of species were Labridae (14), Serranidae (9), Pomacentridae (8), and Haemulidae and Chaetodontidae (5). Out of all the species, $15(17 \%)$ were found in all five localities, $4(4.5 \%)$ were exclusive from Morro and Remanso, 8 (9.1\%) from Isla Aguja, 9 (10.2\%) from Chengue and $3(3.4 \%)$ from Cinto. The greatest number of species and the greatest richness, uniformity and diversity were found in Isla Aguja. Although Chengue and Cinto bays had a similar number of species ( 50 and 49 , respectively), there was a greater diversity $\left(H^{\prime}=3.33\right)$ and uniformity $(\mathrm{J}=0.85)$ in Chengue. Morro and Remanso showed similar values in the three indexes (Table 1).

Of the twenty most abundant species in all five localities (\#ind./120 $\mathrm{m}^{2}$ ), those that showed higher values were Thalassoma bifasciatum and Abudefduf saxatilis with 48 and 47 individuals. They were followed by Chromis cyanea, Clepticus parrae, Stegastes partitus, Haemulon aurolineatum, and Canthigaster rostrata with abundances between 22 and 32 individuals. Pterois volitans was found with an average abundance per transect of 9.8 ind. $/ 120 \mathrm{~m}^{2}$ which place it in 11th place (Figure 1).

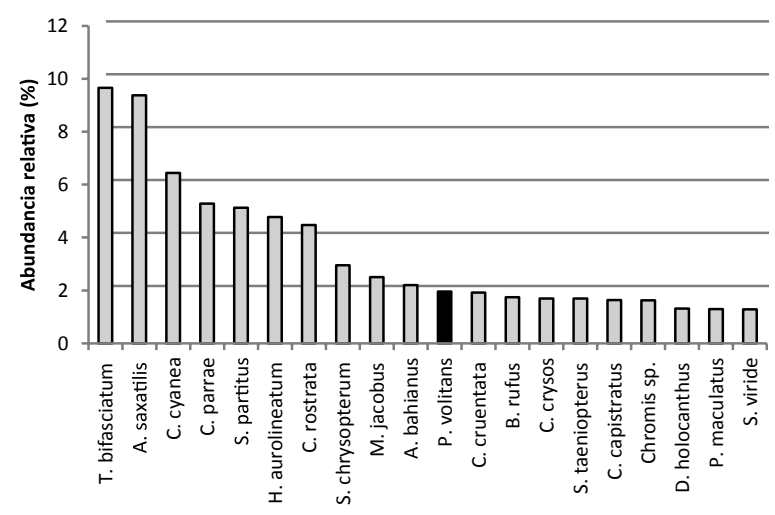

Figure 1. Relative abundance (\#ind./120 m2) of the 20 most representative species.

According to their feeding habits, invertivore species predominated $(45.1 \%)$, the most abundant being $T$. bifasciatum, $C$. rostrata, and $H$. aurolineatum. Carnivore species followed in importance $(24 \%)$; in this case, $P$. volitans was the most abundant species in this group, along with Cephalopholis cruentata. Herbivores came in third place $(18 \%)$, S. partitus and Sparisoma chrysopterum were the most abundant. Lastly, sustrato y la abundancia de la comunidad íctica, se realizó un análisis exploratorio de componentes del sustrato y se determinó la máxima correlación (Bioenv). Posteriormente, se realizó un DistLM (modelo lineal basado en la distancia) con la inclusión de aquellas variables que presentaron los coeficientes más altos; esta prueba permite describir los modelos entre la estructura de la comunidad íctica usando las variables ambientales de componentes del sustrato que describe correlaciones significativas. Los análisis se realizaron con el programa estadístico Primer 6, versión 6.1.13.

\section{RESULTADOS}

En total se identificaron 88 especies de peces correspondientes a 32 familias (Tabla 1). De éstas, las que presentaron el mayor número de especies fueron: Labridae (14), Serranidae (9), Pomacentridae (8), Haemulidae (5) y Chaetodontidae (5). Del total de especies, 15 $(17 \%)$ fueron comunes a las cinco localidades, 4 (4.5\%) fueron exclusivas para Morro y Remanso, $8(9.1 \%)$ para Isla Aguja, $9(10.2 \%)$ para Chengue y $3(3.4 \%)$ para Cinto. En Isla Aguja se encontró el mayor número de especies y las más altas riqueza, uniformidad y diversidad. A pesar que las bahías de Chengue y Cinto tuvieron un número similar de especies $(50$ y 49 , respectivamente) hubo una mayor diversidad $\left(H^{\prime}=3.33\right)$ y uniformidad $(J=0.85)$ en Chengue. Morro y Remanso presentaron valores similares para los tres índices (Tabla 1 ).

Entre las veinte especies más abundantes para las cinco localidades (\#ind./120 $\mathrm{m}^{2}$ ), las que tuvieron mayores valores fueron Thalassoma bifasciatum y Abudefduf saxatilis con 48 y 47 individuos. Le siguieron Chromis cyanea, Clepticus parrae, Stegastes partitus, Haemulon aurolineatum y Canthigaster rostrata con abundancias entre 22 y 32 individuos. Pterois volitans se encontró con una abundancia promedio por transecto de 9.8 ind./120 $\mathrm{m}^{2}$ ocupando el undécimo lugar (Figura 1).

De acuerdo con sus hábitos alimentarios predominaron las especies invertívoras (45.1\%), siendo las más abundantes $T$. bifasciatum, C. rostrata y $\mathrm{H}$. aurolineatum. Las especies carnívoras siguieron en importancia (24\%); en este caso $P$. volitans fue la especie más abundante de este grupo junto con Cephalopholis cruentata. Los herbívoros se ubicaron en tercer lugar $(18 \%)$, siendo S. partitus y Sparisoma chrysopterum las más abundantes. Por último aparecen las planctívoras $(12.1 \%)$ con $A$. saxatilis y C. cyanea. Entre localidades se encontró en 
Table 1. Relative abundance of each species, total number of species, richness (d) equitability indexes (J) Shannon-Weaver diversity $\left(H^{\prime}\right)$. M: Morro; R: Remanso; IA: Isla Aguja; $\mathrm{CH}$ : Bay of Chengue and C: Bay of Cinto. The most representative families are in bold letters.

\begin{tabular}{|c|c|c|c|c|c|c|}
\hline \multirow{2}{*}{ FAMILIES/SPECIES } & \multirow{2}{*}{ HAB. ALIM } & \multicolumn{5}{|c|}{ Locality } \\
\hline & & $\mathbf{M}$ & $\mathbf{R}$ & IA & $\mathrm{CH}$ & C \\
\hline \multicolumn{7}{|l|}{ ACANTHURIDAE } \\
\hline Acanthurus bahianus & Herb & 1.18 & 1.96 & 2.67 & 3.30 & 1.87 \\
\hline A. coeruleus & Herb & & 0.59 & 1.34 & 2.20 & 1.87 \\
\hline A. chirurgus & Herb & & 0.20 & 1.34 & & 0.94 \\
\hline \multicolumn{7}{|l|}{ AULOSTOMIDAE } \\
\hline Aulostomus maculatus & Carn & 0.95 & 0.98 & 2.67 & 1.10 & 0.37 \\
\hline \multicolumn{7}{|l|}{ BALISTIDAE } \\
\hline Balistes vetula & Invert & & & & 0.22 & \\
\hline \multicolumn{7}{|l|}{ BOTHIDAE } \\
\hline Bothus lunatus & Carn & & & 0.27 & & \\
\hline \multicolumn{7}{|l|}{ CARANGIDAE } \\
\hline Caranx crysos & Carn & 2.37 & & 5.35 & & 0.75 \\
\hline C. ruber & Carn & 1.18 & & & & \\
\hline Elagatis bipinnulata & Carn & & & & & 1.12 \\
\hline \multicolumn{7}{|l|}{ CHAETODONTIDAE } \\
\hline Chaetodon striatus & Invert & 0.47 & 0.39 & & 1.32 & 0.37 \\
\hline C. capistratus & Invert & 0.47 & 4.91 & 1.07 & 0.44 & 1.31 \\
\hline C. sedentarius & Invert & 0.47 & & & & 0.75 \\
\hline C. ocellatus & Invert & 1.42 & 1.57 & 2.14 & 0.44 & 0.56 \\
\hline Prognathodes aculeatus & Invert & 0.24 & 0.98 & 1.34 & & \\
\hline \multicolumn{7}{|l|}{ DIODONTIDAE } \\
\hline Diodon holocanthus & Invert & 0.95 & 1.18 & 1.60 & 2.64 & 0.19 \\
\hline \multicolumn{7}{|l|}{ CIRRHITIDAE } \\
\hline Amblycirrhitus pinos & Invert & 0.24 & & & & \\
\hline \multicolumn{7}{|l|}{ EPHIPPIDAE } \\
\hline Chaetodipterus faber & Invert & & 0.20 & 1.87 & 0.44 & 0.19 \\
\hline \multicolumn{7}{|l|}{ FISTULARIDAE } \\
\hline Fistularia tabacaria & Carn & & 0.20 & & & \\
\hline \multicolumn{7}{|l|}{ HAEMULIDAE } \\
\hline Anisotremus virginicus & Invert & & & & 2.42 & 1.87 \\
\hline A. surinamensis & Invert & & & 0.27 & & \\
\hline Haemulon aurolineatum & Invert & & & & 21.98 & 1.87 \\
\hline H. flavolineatum & Invert & & 1.96 & 0.27 & 1.10 & \\
\hline H. plumierii & Invert & & 0.98 & 0.27 & 3.30 & 0.19 \\
\hline \multicolumn{7}{|l|}{ HOLOCENTRIDAE } \\
\hline Myripristis jacobus & Plact & & 3.93 & 8.02 & & 0.56 \\
\hline Holocentrus rufus & Plact & & 0.59 & 2.67 & & 0.37 \\
\hline \multicolumn{7}{|l|}{ LABRIDAE } \\
\hline Clepticus parrae & Plact & 2.84 & & 4.81 & & 18.73 \\
\hline Bodianus rufus & Invert & 1.66 & 0.59 & 0.53 & 2.20 & 3.75 \\
\hline Halichoeres garnoti & Invert & 0.47 & 0.59 & 2.67 & & \\
\hline H. bivittatus & Invert & & 0.20 & & & \\
\hline Thalassoma bifasciatum & Invert & 2.37 & 19.65 & 5.35 & 2.20 & 18.73 \\
\hline Scarus iseri & Herb & & 1.96 & & & 1.31 \\
\hline S. taeniopterus & Herb & 4.74 & & 1.34 & 2.20 & 0.19 \\
\hline S. guacamaia & Herb & & & & 0.44 & \\
\hline S. vetula & Herb & & & & 2.20 & \\
\hline Sparisoma chrysopterum & Herb & 11.85 & 1.96 & & & 0.94 \\
\hline S. aurofrenatum & Herb & & & & 0.88 & \\
\hline S. viride & Herb & 1.90 & 1.96 & 0.27 & 0.44 & 1.87 \\
\hline S. atomarium & Herb & 0.24 & 0.20 & & & \\
\hline S. rubripinne & Herb & & 1.96 & & & \\
\hline LUTJANIDAE & & & & & & \\
\hline Lutjanus apodus & Carn & & & & 0.88 & \\
\hline L. cyanopterus & Carn & & & & 1.76 & \\
\hline L. mahogoni & Carn & & & 0.53 & 4.40 & 0.56 \\
\hline Ocyurus chrysurus & Carn & & & & 4.40 & 0.19 \\
\hline MALACANTHIDAE & & & & & & \\
\hline
\end{tabular}




\begin{tabular}{|c|c|c|c|c|c|c|c|}
\hline Malacanthus plumieri & Carn & & & & 0.27 & & \\
\hline \multicolumn{8}{|l|}{ MONACANTHIDAE } \\
\hline Aluterus scriptus & Invert & 0.24 & & & 0.27 & & 0.19 \\
\hline Cantherhines macrocerus & Invert & 0.24 & & & 0.27 & & \\
\hline C. pullus & Invert & 0.47 & & & 0.27 & & 0.19 \\
\hline \multicolumn{8}{|l|}{ MULLIDAE } \\
\hline Pseudupeneus maculatus & Invert & 0.71 & 1.96 & & 0.53 & 3.30 & \\
\hline Mulloidichthys martinicus & Invert & & 1.96 & & 2.67 & 0.88 & \\
\hline \multicolumn{8}{|l|}{ MURAENIDAE } \\
\hline Gymnothorax moringa & Carn & & & & 0.80 & 0.44 & 0.37 \\
\hline G. miliaris & Carn & & & & 0.27 & & \\
\hline \multicolumn{8}{|l|}{ OSTRACIIDAE } \\
\hline Lactophrys triqueter & Invert & 0.24 & 0.59 & & 1.07 & 1.10 & 0.75 \\
\hline L. bicaudalis & Invert & & & & & & 0.37 \\
\hline Acanthostracion polygonius & Invert & & 0.20 & & 0.53 & 0.66 & \\
\hline \multicolumn{8}{|l|}{ OPISTOGNATHIDAE } \\
\hline Opistognathus aurifrons & Plact & & & & 2.67 & & 0.94 \\
\hline \multicolumn{8}{|l|}{ PRIACANTHIDAE } \\
\hline Heteropriacanthus cruentatus & Plact & 1.42 & & & 0.27 & & 0.19 \\
\hline \multicolumn{8}{|l|}{ OPHICHTHIDAE } \\
\hline Ophichthus ophis & Carn & 0.24 & & & & & 0.19 \\
\hline \multicolumn{8}{|l|}{ POMACANTHIDAE } \\
\hline Holacanthus tricolor & Invert & 0.47 & 0.98 & & 0.53 & 1.10 & 0.75 \\
\hline H. ciliaris & Invert & & 0.98 & & 0.27 & & \\
\hline Pomacanthus paru & Invert & & & & 0.53 & 1.10 & \\
\hline P. arcuatus & Invert & & & & 0.27 & 0.22 & \\
\hline \multicolumn{8}{|l|}{ POMACENTRIDAE } \\
\hline Abudefduf saxatilis & Plact & 1.42 & 19.65 & & 2.67 & 4.40 & 18.73 \\
\hline Chromis cyanea & Plact & 23.70 & 0.98 & & 5.35 & 2.20 & \\
\hline C. multilineata & Plact & 1.18 & 5.89 & & & 0.66 & \\
\hline C. insolata & Plact & 1.90 & & & 6.68 & & \\
\hline Stegastes partitus & Herb & 16.59 & & & 5.35 & 2.20 & 1.50 \\
\hline S. adustus & Herb & & & & & 5.49 & \\
\hline S. planifrons & Herb & & & & 1.34 & & \\
\hline Microspathodon chrysurus & Herb & 1.42 & 0.98 & & 0.80 & 0.44 & \\
\hline \multicolumn{8}{|l|}{ SCIAENIDAE } \\
\hline Equetus punctatus & Invert & 0.47 & & & & 0.44 & 0.19 \\
\hline Pareques acuminatus & Invert & & & & & 0.44 & \\
\hline \multicolumn{8}{|l|}{ SCOMBRIDAE } \\
\hline Auxis thazard & Carn & 0 & & 0 & 2.67 & 0 & 2.89 \\
\hline \multicolumn{8}{|l|}{ SCORPAENIDAE } \\
\hline Pterois volitans & Carn & 2.84 & 0.98 & & 1.87 & 2.42 & 1.69 \\
\hline Scorpaena plumieri & Carn & & & & 0.27 & & \\
\hline \multicolumn{8}{|l|}{ SERRANIDAE } \\
\hline Hypoplectrus nigricans & Carn & 0.24 & & & & 2.20 & 0.94 \\
\hline H. guttavarius & Carn & & & & & & 0.19 \\
\hline H. puella & Carn & 2.37 & & & 0.27 & 2.20 & \\
\hline H. unicolor & Carn & 0.24 & & & & 0.66 & 0.94 \\
\hline Mycteroperca $s p$ & Carn & & & & & 0.22 & \\
\hline Cephalopholis cruentata & Carn & 2.13 & 3.93 & & 2.67 & 0.66 & 0.19 \\
\hline C. fulvus & Carn & & & & 0.27 & & 0.37 \\
\hline Serranus tigrinus & Invert & 1.18 & 2.95 & & 0.80 & 0.22 & 0.94 \\
\hline Rypticus saponaceus & Carn & & & & 0.80 & & \\
\hline SPHYRAENIDAE & & & & & & & \\
\hline Sphyraena barracuda & Carn & & & & & 1.10 & 0.19 \\
\hline SYNGNATHIDAE & & & & & & & \\
\hline Hippocampus reidi & Plact & & 0.20 & & & & \\
\hline SYNODONTIDAE & & & & & & & \\
\hline Synodus intermedius & Carn & 0.47 & 0.20 & & & 1.76 & 0.94 \\
\hline TETRAODONTIDAE & & & & & & & \\
\hline Canthigaster rostrata & Invert & 3.55 & 7.86 & & 8.02 & 0.66 & 2.25 \\
\hline Sphoeroides spengleri & Invert & 0.24 & & & & & \\
\hline Número de especies & & 43 & 40 & & 54 & 50 & 49 \\
\hline J & & 0.76 & 0.78 & & 0.87 & 0.85 & 0.72 \\
\hline $\mathrm{H}^{\prime}$ & & 2.85 & 2.88 & & 3.49 & 3.33 & 2.80 \\
\hline
\end{tabular}


planktivores (12.1\%) with $A$. saxatilis and $C$. cyanea. As for localities, Remanso presented the greatest percentage of invertivore, herbivore, and planktivore groups $(50 \%, 22.5 \%$, and $15 \%$ respectively). Carnivores were the most abundant in Cinto bay (32.7\%) (Figure 2).

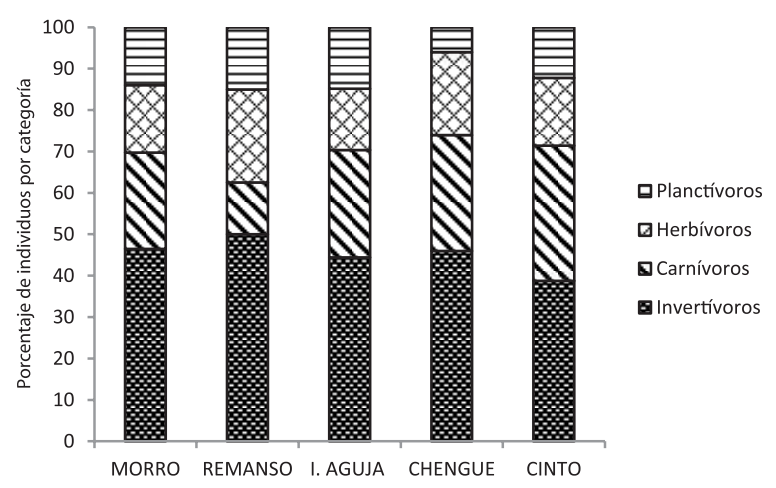

Figure 2. Percentage breakdown of trophic groups in each locality.

Relating to substrate, a greater percentage of coral coverage was found in Chengue $(70 \%)$, followed by Cinto (27\%), and Isla Aguja (24\%). The presence of gorgonias was higher in Remanso $(37 \%)$ and Morro $(32 \%)$. In Cinto macroalgae predominated $(47 \%)$; and the abiotic substrate was greater in Remanso (41\%) and Isla Aguja $(37 \%)$. The rest of the components were variable for each locality with percentages greater than $15 \%$ (Figure 3 ).

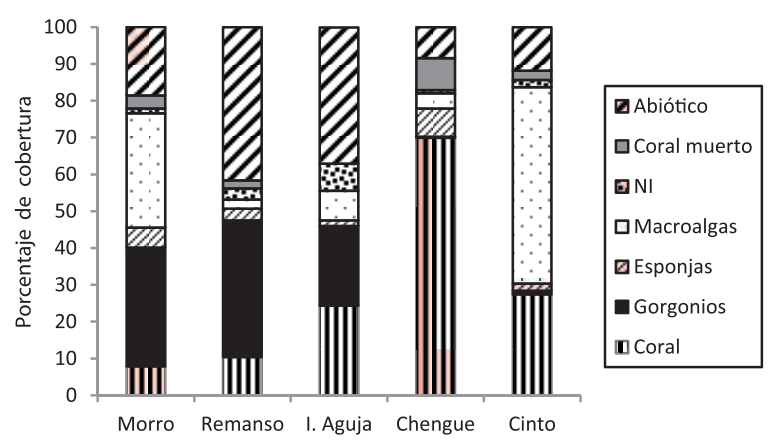

Figure 3. Percentage breakdown of the main substrate components in each locality.

The fish community in Chenque bay is distinct from the rest of the reef formations both in the exploratory analysis (Bioenv, DistLM) (Figure 4) as well as in the Bray-Curtis dendrogram of similarity. Groups that were defined as similar were in Morro and Isla Aguja (55\%) and Remanso and Cinto (52\%) (Figure 5). Corals, gorgonias and sponges were the substrate component variables that best related to the abundance in
Remanso el mayor porcentaje de los grupos de invertívoros, herbívoros y planctívoros (50, 22.5 y $15 \%$ respectivamente). Los carnívoros para la bahía de Cinto fueron los más abundantes (32.7\%) (Figura 2).

Con respecto al sustrato, se encontró el mayor porcentaje de corales en la bahía de Chengue (70\%), seguida por la de Cinto (27\%) e Isla Aguja (24\%). La presencia de gorgonias fue alta en Remanso (37\%) y Morro (32\%). En Cinto predominaron las macroalgas (47\%); y el sustrato abiótico fue mayor en Remanso (41\%) e Isla Aguja (37\%). El resto de componentes fue variable para cada localidad con porcentajes menores a $15 \%$ (Figura 3 ).

La comunidad íctica de la bahía de Chenque se separa del resto de las formaciones arrecifales evaluadas tanto con los análisis exploratorios (Bioenv, DistLM) (Figura 4) como con el dendrograma de similaridad de Bray-Curtis. Donde los grupos que se definieron como cercanos fueron las localidades de Morro e Isla Aguja (55\%) y Remanso y Cinto (52\%) (Figura 5). La cobertura de corales, gorgonias y esponjas fueron las variables de los componentes de sustrato que mejor se relacionaron con la abundancia de la comunidad íctica, con una correlación de 0.806 . El 33\% de la variación se explica para la bahía de Chengue con la asociación de corales y esponjas, mientras que en Morro y Remanso con el sustrato abiótico (Figura 4).

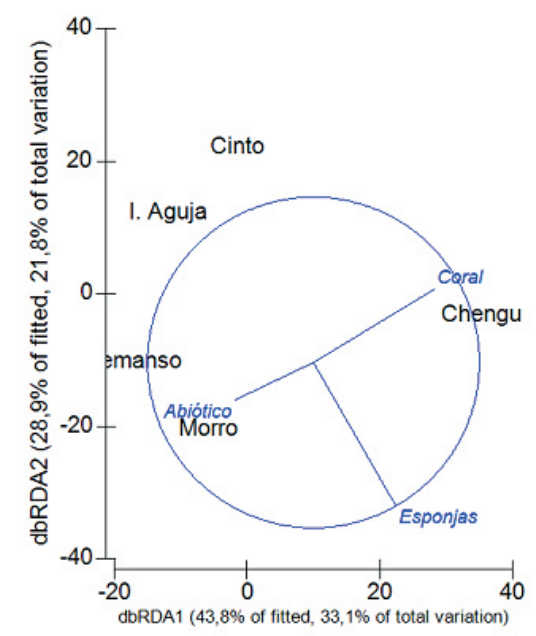

Figure 4. Multidimensional scale between fish species per locality and the coverage of benthic organisms. 


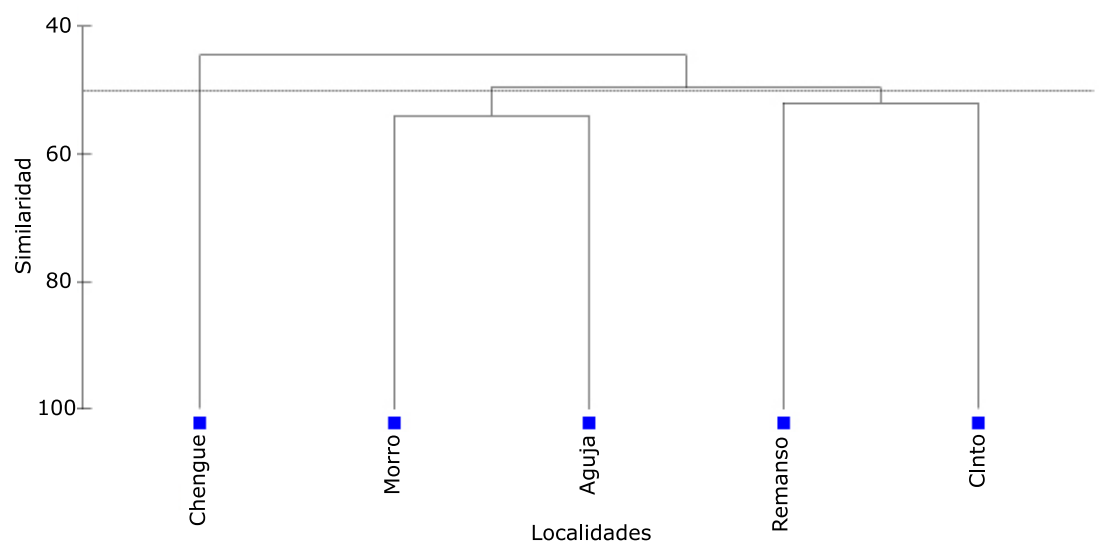

Figure 5. Group analysis (Bray-Curtis) of the relative abundance of fish communities between localities.

the fish community, with a correlation of 0.806 . $33 \%$ of the variation is explained in Chengue by associating corals and sponges, while in Morro and Remanso with abiotic substrate (Figure 4).

\section{DISCUSSION}

For Gaira bay ( $3 \mathrm{~km}$ southeast of the Santa Marta bay) Grijalba-Bendeck et al (18) evaluated fish through the technique of stationary visual census and the results showed that families Serranidae, Lutjanidae, Labridae and Scaridae [(presently included in Labridae (19)] were the most abundant. Evaluating fish associated to coral formations in the Caribbean region of Colombia, specifically Chengue, Reyes-Nivia et al (20) showed the importance of families Serranidae, Scaridae [(presently included in Labridae (19)], Labridae, Pomacentridae, Haemulidae, Lutjanidae, and Gobiidae; Olaya-Restrepo et al (21) evaluated the catch of reef fishes in 20 stations near Santa Marta and TNP and showed that families Serranidae, Haemulidae, Gobiidae, Scaridae [(presently included in Labridae (19)] and Pomacentridae as the most dominant.

In general terms, and as shown in this study, the abundance of families Serranidae, Haemulidae, and Labridae is constant; however, family Lutjanidae, that was believed important did not appear as such in Olaya-Restrepo et al (21). Nevertheless, family Gobiidae, which is usually well represented, was not recorded; this could be due to the methods and purposes previously used, since, in this case, cracks and rifts were not examined during the census, and large areas and different depth strata were not covered.

Regarding species numbers, Acero and Garzón (1) list 372 species from the Santa Marta region, considering it as being biologically rich. This

\section{DISCUSIÓN}

Para el morro de la bahía de Gaira $(3 \mathrm{~km}$ al suroccidente de la bahía de Santa Marta) GrijalbaBendeck et al (18) realizaron la evaluación de peces a través de la técnica de censo visual estacionario y sus resultados muestran a las familias Serranidae, Lutjanidae, Labridae y Scaridae [(incluida actualmente en Labridae (19)] como las más abundantes. Al evaluar los peces asociados a formaciones coralinas del Caribe colombiano, específicamente para la bahía de Chengue, Reyes-Nivia et al (20) destacan la importancia de las familias Serranidae, Scaridae [(incluida actualmente en Labridae (19)], Labridae, Pomacentridae, Haemulidae, Lutjanidae y Gobiidae; Olaya-Restrepo et al (21), evaluaron los ensamblajes de peces arrecifales en 20 estaciones del área de Santa Marta y el PNNT y mostraron a las familias Serranidae, Haemulidae, Gobiidae, Scaridae [(incluida actualmente en Labridae (19)] y Pomacentridae como las más dominantes.

En términos generales y al igual con lo obtenido en este estudio, persisten en su abundancia familias como Serranidae, Haemulidae y Labridae; sin embargo, se destaca que la familia Lutjanidae, que inicialmente se marcaba como importante, no apareció como tal en el estudio de Olaya-Restrepo et al (21). La familia Gobiidae, por el contrario, aunque usualmente está bien representada, no fue registrada; esto puede ser resultado de los métodos y propósitos previos pues, en este caso, no se examinaron grietas o hendiduras durante los censos y no se cubrieron áreas tan extensas, ni diferentes estratos de profundidad.

Con respecto al número de especies, Acero y Garzón (1) listan para la región de Santa Marta 372 , enmarcando a la región como de una gran riqueza biológica. Documento que se constituye en un punto de partida para comparaciones con 
document is a starting point for comparing the present state of the fish fauna in the region. For example, Grijalba-Bendeck et al (18) mentioned that $30 \%$ of the previously mentioned species were recorded in Gaira; additionally, those belonging to the Serranidae family were found in reduced numbers, which the authors attributed possibly to overfishing. Reyes-Nivia et al (20) list 149 species in Chengue, with commercially important fish of the families Serranidae and Lutjanidae, such as Mycteroperca acutirostris, $M$. bonaci, M. interstitialis, M. tigris, M. venenosa, Lutjanus analis, L. griseus, and $L$. jocu, none of which were seen in this study.

Chengue bay, where mangrove, marine grass and reef patches are present but were not evaluated because reef formation monitoring was already established in its central area; the species listed (50 species) were less than those found by OlayaRestrepo et al (21) (between 62 and 95 species). However, Lutjanus cyanopterus was recorded, a piscivorous species that could play an important role in the control of lionfish, even more so since, as previously mentioned, no other species that could compete or prey on the invader was seen.

Olaya-Restrepo et al (21) studied 20 stations showing an average diversity index of 1.73 in TNP and 1.68 in unprotected areas; four localities coincide with this study and the results generally show a greater number of species (70 in Morro, 62 in Isla Aguja, 62 and 95 in Chengue and between 70 and 80 in Cinto), although commercially important fish such as Mycteroperca tigris, $M$. venenosa, M. phenax, Epinephelus guttatus, $E$. itajara, Lutjanus analis and L. buccanella were not recorded. Additionally lower averages for individuals and much lower densities were found in large snapper and grouper species; only $L$. mahogoni was abundant in the TNP protected area and Cephalopholis cruentata in unprotected stations.

In comparison with that study, L. mahogoni, in spite of being observed in the park, only had a relevant abundance in Chengue, and $C$. cruentata, which was present in the five localities, was more abundant in those outside of TNP. In spite of being considered a protected area, Isla Aguja is visited daily by tourists and fishermen; it was the reef formation that had the greatest richness and diversity of species ( 54 species and $\mathrm{H}^{\prime}=3.49$ ) and that, along with Chengue and Cinto, presented the highest diversity values. Krajewski and Floeter (17) mentioned the protected marine areas as an important factor that can influence the differences in fish communities. Their study compared protected and uninhabited fishing sites with a marine park that permits local fishing, and el estado actual de la ictiofauna en la región. Por ejemplo, Grijalba-Bendeck et al (18) mencionan que para Gaira se registró un $30 \%$ de las especies previamente citadas; además, se encontró con abundancias bajas a todas aquellas pertenecientes a la familia Serranidae, atribuida esta disminución por los autores a una posible explotación pesquera. Reyes-Nivia et al (20) listan para Chengue 149 especies, con peces de importancia comercial de las familias Serranidae y Lutjanidae como Mycteroperca acutirostris, M bonaci, M. interstitialis, M. tigris, M. venenosa, Lutjanus analis, L. griseus y $L$. jocu, ninguna de las cuales fue vista en este estudio.

La Bahía de Chengue, una localidad con manglar, pastos marinos y varios parches arrecifales, sitios que no fueron evaluados debido a que la formación arrecifal de seguimiento se estableció en su parte central; de allí que las especies listadas (50 especies) fueran menos que las encontradas por Olaya-Restrepo et al (21) (entre 62 y 95 especies). Sin embargo, sí fue registrada Lutjanus cyanopterus, una especie piscívora que podría estar desempeñando un papel importante en el control del pez león, más aun cuando, como se mencionó anteriormente, no fue vista ninguna otra de las especies mencionadas que pudieran competir o depredar al invasor.

El estudio de Olaya-Restrepo et al (21) para 20 estaciones mostró un índice de diversidad promedio de 1.73 en el PNNT y 1.68 en áreas sin protección; cuatro localidades coinciden con este estudio y sus resultados muestran en general un mayor número de especies (70 en Morro, 62 en Isla Aguja, 62 y 95 para Chengue y entre 70 y 80 para Cinto), aunque peces de importancia comercial como Mycteroperca tigris, M. venenosa, M. phenax, Epinephelus guttatus, E. itajara, Lutjanus analis y $L$. buccanella no fueron registrados. Además, abundancias promedio menores a un individuo y densidades muy bajas fueron encontradas para especies de pargos y meros de gran tamaño; solamente L. mahogoni fue abundante en el área protegida del PNNT y Cephalopholis cruentata en estaciones sin protección.

Lo anterior, en comparación con ese estudio, $L$. mahogoni, a pesar de que fue observada en las localidades del parque, su abundancia solo fue relevante para Chengue, y $C$. cruentata, que se presentó en las cinco localidades, fue más abundante para aquellas fuera del PNNT. Isla Aguja a pesar de estar delimitada como área protegida, es visitada diariamente por turistas y pescadores; fue la formación arrecifal con la mayor riqueza y diversidad de especies (54 especies y $\mathrm{H}^{\prime}=3.49$ ) y que, junto con las bahías de Chengue y Cinto, presentó los valores de diversidad más altos. 
it was found that the main species that interest fishermen were the macrocarnivores, so that the great predatory fish in the trophic chain were less abundant. In the Santa Marta region, it has been determined that important carnivore species have been diminishing $(18,20,21)$, which could have had an effect on the proliferation of the invading species.

The structure of the fish community is due to biotic variables such as the abundance of potential competitors (22) and prey (15). In this case, with the presence of the invader, Maljkovic et al (23) found that in the Bahamas M. tigris and $E$. striatus preyed on the lionfish. Mumby et al (24), in the same region, studied a group of groupers, such as E. striatus, M. tigris, M. bonaci, $M$. venenosa and $M$. interstitialis, founding a seven fold lineal reduction in the lionfish biomass in relation to groupers; therefore, evidence suggests that predators can play a role as natural control. In this study, the rarity or absence of mid-sized piscivore can impact the proliferation of the invading species, which is the most abundant predatory fish, even above the small carnivores that are not actively sought by fishermen; this hypothesis should be evaluated in future studies.

In terms of species composition in reef formations where lionfish is present, Lesser and Slattery (25) studied a reef in the Bahamas between 2003 and 2009 with phase changes in communities at mesophotic depths in which, as herbivore fish diminished due to the presence of lionfish, the areas became dominated by algae. The study shows how, under invader presence and its depredation, 19 species were utilized with the consequent reduction of herbivore, planktivore, and spongivore species as well as those that feed on small crustaceans. This impact is called cascade, whether directly due to the consumption of herbivores or indirectly due to species displacement.

The study performed by Morris and Akins (26) mentioned that the lionfish diet is diverse, feeding mainly on small fish that are equally important for many commercially important species such as Serranidae and Lutjanidae. Regarding the analysis of the stomach content of lionfish in North Carolina, Muñoz et al (27) suggested a general carnivore diet made up of $3.8 \%$ crustaceans, cephalopods, bivalves and ophiuroids, and the rest composed by 16 fish families, the most abundant being Haemulidae, Labridae, and Serranidae. In the Colombian Caribbean, lionfish diet was initially analyzed by Muñoz-Escobar and Gil-Agudelo (28) and it was likewise identified as a generalist carnivore, with the family Serranidae being the most
Krajewski y Floeter (17) mencionan a las áreas marinas protegidas como un factor importante que puede influir en las diferencias de la comunidad de peces. Su estudio comparó sitios protegidos de la pesca y no habitados, con un parque marino pero con pesca local permitida; se encontró que las principales especies de interés por los pescadores fueron macrocarnivoros, haciendo así que los peces grandes depredadores de la cadena trófica, fueran especies menos abundantes. Para la región de Santa Marta se ha determinado la disminución de especies carnívoras importantes $(18,20,21)$, lo cual puede haber favorecido la proliferación de la especie invasora.

La estructura de la comunidad de peces responde a variables bióticas, tales como la abundancia de competidores potenciales (22) y de presas (15). Para el caso actual con la presencia del invasor, luego que Maljkovic et al (23) encontraran en las Bahamas a especies como $M$. tigris y $E$. striatus conteniendo como presa al pez león, Mumby et al (24), en la misma región, estudiaron un grupo de meros y chernas tales como $E$. striatus, $M$. tigris, $M$. bonaci, M. venenosa y $M$. interstitialis, encontrando una reducción lineal de siete veces en la biomasa del pez león en relación con los meros y chernas; por consiguiente, la evidencia sugiere que la depredación por estas especies sí puede cumplir la función de control natural. En el caso de este trabajo, la notoria rareza o ausencia de depredadores piscívoros de mediano tamaño puede incidir sobre la proliferación de la especie invasora, que ya es la más abundante de los peces depredadores, por encima incluso de los pequeños carnívoros que no son buscados activamente por los pescadores; esta hipótesis debe ser evaluada en futuros estudios.

En términos de la composición de las especies en las formaciones arrecifales con la presencia del pez león, para un arrecife en las Bahamas entre 2003 y 2009, Lesser y Slattery (25) estudiaron cambios de fase de comunidades a profundidades mesofóticas, donde con la disminución de peces herbívoros con la presencia del pez león, pasaron a estar dominadas por algas. El estudio muestra cómo, con la presencia del invasor y su depredación, se dio una disminución de 19 especies y la consecuente reducción de peces herbívoros, planctívoros, espongívoros y los que se alimentan de pequeños crustáceos. Impacto denominado de cascada, ya sea directamente por el consumo de herbívoros o indirecto por el desplazamiento de especies.

El estudio de Morris y Akins (26) menciona que la dieta del pez león es diversa alimentándose principalmente de peces pequeños, que son igualmente importantes para muchas especies de importancia comercial, como serránidos 
predominant. Also, species of the families Gobiidae, Clupeidae, and Pomacentridae that, although have little or no commercial importance, are part of the diet of Lutjanidae and Haemulidae, which are commercial families that have diminished their abundances in recent years.

Muñoz et al (27) mentioned that the lionfish diet would be determined according to prey availability. In our study, although the results showed that carnivore, invertivore and herbivore species were predominant, they had low abundances. Thus, in analyzing the 20 most abundant species, planktivores and invertivores were better represented. More than half $(70 \%)$ of the fish abundance in the Santa Marta region was composed of ten species. Of these, three are invertivores, four are planktivores, and three are herbivores, ingesting plankton and small invertebrate and algae; those ten species are smaller than the lionfish and, due to their abundance, could have become prey too and influenced the proliferation of the invader (28). It is clearly indicative of the overexploitation of fish resources in the Santa Marta region that the invader quickly became one of the most abundant species and the most important fish predators.

Whitfield et al (29) compared the abundance of lionfish with groupers between 35-50 m deep and between 50 and $100 \mathrm{~m}$ in North Carolina. Lionfish was found with average abundance at $21.1 \mathrm{ind} / \mathrm{ha}$ in shallow areas and $5.2 \mathrm{ind} / \mathrm{ha}$ in deep waters, and in both depths it was observed second in abundance, after Mycteroperca phenax. Unfortunately, there is no starting point recorded in 2008 and 2009, which is when lionfish appeared in Colombian waters $(6,12)$. In general, no records show the Scorpaenidae family as significant in abundance or biomass in the Colombian Caribbean. Now, under lionfish presence, it was observed to be even more important than species such as $C$. cruentata, which was the only one observed among the twenty most abundant species and no grouper of considerable size was observed in the sampled sites that could act as a natural control for the invader.

According to Munday et al (30) and Gardiner and Jones (31), some types of sustrate, such as corals and sponges, can be used as feeding sites or refuge, and these can influence fish community structure. For example, strong correlations have been found between substrate composition and reef fish with specialized diets densities (e.g. butterflyfish and angelfish) and with habitat requirements (gobies and some cardinalfish) (15). When analyzing density of fish biomass, Krajewski and Floeter (17) found y lutjánidos. Sobre el análisis del contenido estomacal del pez león en Carolina del Norte, Muñoz et al (27) sugieren una dieta carnívora generalista conformada en $3.8 \%$ por crustáceos, cefalópodos, bivalvos y ofiuros, y el resto por 16 familias de peces, con los haemúlidos, scáridos y serránidos como los más abundantes. Para el Caribe colombiano, la dieta del pez león fue analizada preliminarmente por Muñoz-Escobar y Gil-Agudelo (28) e igualmente se le identificó como un carnívoro generalista, con la familia Serranidae como la más predominante. Además especies de las familias Gobiidae, Clupeidae y Pomacentridae que, aunque de poca o ninguna importancia comercial, son parte de la dieta de lutjánidos y haemúlidos, familias comerciales que han registrado bajas abundancias en los últimos años.

Muñoz et al (27) mencionan que la dieta del pez león estaría dada de acuerdo con la disponibilidad de presas. En este estudio, aunque los resultados mostraron la predominancia de especies carnívoras, invertívoras y herbívoras, estas tuvieron bajas abundancias. Es así, como del análisis de las 20 especies más abundantes, fueron las planctívoras e invertívoras las más representativas. Diez especies sumaron más de la mitad (70\%) de la abundancia de peces en la región de Santa Marta. De ellas, tres son invertívoras, cuatro son planctívoras y tres son herbívoras, ingiriendo plancton y pequeños invertebrados y algas; esas diez especies son de menor talla que el pez león y podrían haber favorecido con su abundancia la proliferación del invasor, al constituirse en sus presas principales (28). Es claramente indicativo de la situación de sobreexplotación de los recursos ícticos de la región de Santa Marta que el invasor se haya posicionado con relativa rapidez como una de las especies más abundante y la primera de los peces ictiófagos.

Whitfield et al (29) compararon la abundancia del pez león con la de meros y chernas entre $35-50 \mathrm{~m}$ de profundidad y entre 50 y $100 \mathrm{~m}$ en Carolina del Norte. El pez león se encontró con una abundancia promedio de 21.1 ind/ha en las partes someras y de 5.2 ind/ha en las profundas $y$, a ambas profundidades, fue observado como segundo en abundancia luego de Mycteroperca phenax. Desafortunadamente, no se cuenta con un punto de partida entre 2008 o 2009, cuando se registró la aparición del pez león en aguas colombianas $(6,12)$. En general, para el Caribe colombiano ninguno de los registros marcaba a la familia Scorpaenidae como significativa en términos de abundancia o biomasa. Ahora, con la presencia del pez león, este se observó más importante incluso que especies como $C$. cruentata, que fue la única observada entre las veinte más abundantes y ninguna especie de mero o cherna de tamaño considerable 
a positive correlation with coral coverage, depth and areas protected from waves, where mobile carnivores and invertivores were more influenced by unconsolidated bottoms and substrates, planktivores and omnivores by wave exposure, and herbivores by algae coverage, with the existence of general habits that suggest that the community is dominated by versatile species.

Substrate components such as corals and sponges in Chengue bay, and the abiotic substrate in Morro and Remanso, were the variables that best explained fish composition. Association of lionfish to substrate was only evident in Chengue and Morro, localities where the invader was more abundant. It should be kept in mind that in tropical systems positive relationships between coral coverage and fish density and biomass have been considered as indicators of the complexity of the habitat by offering more shelter for medium-sized fish (between 10 and $40 \mathrm{~cm} \mathrm{LT)} \mathrm{(17);} \mathrm{results} \mathrm{show}$ that, while lionfish was more abundant in areas with greater coral coverage, sponges, or abiotic substrate, it has also occupied many other habitats. However, detailed studies need to be performed that discriminate the availability of food, fish composition by locality, and different associated substrates and ecosystems to determine the possible effects on the community.

\section{Acknowledgements}

To Luis Manjarrés Martínez for his support with statistical analysis. To the proposal funded by Fonciencias and the Universidad del Magdalena "Invasión del pez león Pterois volitans registro de sus abundancias, estructura de tallas y preferencia al sustrato en Santa Marta, Caribe colombiano," and to the proposal "Invasión del pez león a los arrecifes del Caribe colombiano: biología, ecología y origen" funded by Colciencias (1361-521-28271). se observó para los sitios muestreados y que pudieran estar contribuyendo como un control natural del invasor.

Según Munday et al (30) y Gardiner y Jones (31), algunos tipos de sustrato, como corales y esponjas, pueden ser usados como sitios de alimentación o refugio y éstos pueden influenciar la estructura de la comunidad de peces. Por ejemplo, correlaciones fuertes se han encontrado entre la composición del sustrato y la densidad de peces arrecifales con dietas especializadas (ej. mariposas y peces ángel) y con requerimientos de hábitat (gobios y algunos cardenales) (15). Al analizar la densidad y biomasa de peces, Krajewski y Floeter (17) hallaron una correlación positiva con la cobertura de coral y la profundidad y negativa con la exposición al oleaje, donde carnívoros e invertívoros móviles estuvieron más influenciados por la profundidad y el substrato no consolidado; planctívoros y omnívoros por la exposición al oleaje y los herbívoros por la cobertura algal, con la existencia de hábitos generalistas que sugieren que la comunidad es dominada por especies versátiles.

Los componentes del sustrato como corales y esponjas para la bahía de Chengue y el sustrato abiótico para Morro y Remanso fueron las variables que mejor explicaron la composición de peces. Para la asociación con el pez león y el sustrato, solo fue evidente para Chengue y Morro, al ser las localidades con mayor abundancia del invasor. Si se tiene en cuenta que en sistemas tropicales, relaciones positivas entre la cobertura de coral y la densidad de peces y la biomasa han sido consideradas como indicadoras de la complejidad del hábitat al ofrecer más refugios a peces de mediano tamaño (entre 10 y $40 \mathrm{~cm} \mathrm{LT)} \mathrm{(17),}$ los resultados mostraron que, si bien el pez león fue más abundante en sitios con mayor cobertura coralina, de esponjas o sustrato abiótico, éste ha ocupado indistintamente cualquier hábitat. Sin embargo, se requieren estudios detallados que discriminen la disponibilidad de alimento, la composición íctica por localidad y los distintos sustratos y ecosistemas asociados para determinar los posibles efectos sobre la comunidad.

\section{Agradecimientos}

A Luis Manjarrés Martínez, por su apoyo en el análisis estadístico. Al proyecto financiado por Fonciencias y la Universidad del Magdalena "Invasión del pez león Pterois volitans registro de sus abundancias, estructura de tallas y preferencia al sustrato en Santa Marta, Caribe colombiano", y al proyecto "Invasión del pez león a los arrecifes del Caribe colombiano: biología, ecología y origen" financiado por Colciencias (1361-521-28271). 


\section{REFERENCES}

1. Acero PA, Garzón J. Peces arrecifales de la región de Santa Marta (Caribe colombiano). I. Lista de especies y comentarios generales. Acta Biol Colom 1987; 1(3):83-105.

2. Acero PA. Una nueva mirada a los peces comerciales del Caribe continental colombiano. An Inst Inv Mar Punta Betín 2003; 22:129-135.

3. Grijalba-Bendeck M, Novoa-Pabón AM, Bustos-Montes DM, Posada-Peláez C, Santafé-Muñoz AM. La pesca artesanal marina del Departamento del Magdalena, Colombia: manejo y conservación. Revista Mutis 2012; 2(2):1-25.

4. Álvarez-León R, Orozco-Rey R, PáramoFonseca M, Restrepo-Santamaría D. Lista de los peces fósiles y actuales de Colombia. Nombres científicos válidos, distribución geográfica, diagnosis de referencia y nombres comunes e indígenas. Bogotá, Colombia: Eco Prints, Diseño gráfico y audiovisual Ltda; 2013.

5. Albins $M$, Hixon $M$. Invasive Indo-Pacific lionfish Pterois volitans reduce recruitment of Atlantic coral-reef fishes. Mar Ecol Prog Ser 2008; 367:233-238.

6. Schofield PJ. Geographic extent and chronology of the invasion of non-native lionfish (Pterois volitans [Linnaeus 1758] and $P$. miles [Bennett 1828]) in the western North Atlantic and Caribbean Sea. Aq Inv 2009; 4(3):473-479

7. Darling E, Green S, Leary J, Coté I. IndoPacific lionfish are larger and more abundant on invaded reefs: a comparison of Kenyan and Bahamian lionfish populations. Biol Invasions 2011; 13:2045-2051.

8. Kulbicki M, Beets J, Chabanet P, Cure K, Darling $E$, Floeter SR, et al. Distributions of Indo-Pacific lionfishes Pterois spp. in their native ranges: implications for the Atlantic invasion. Mar Ecol Prog Ser 2012; 446:189-205.

9. Albins $M$, Hixon $M$. Worst case scenario: potential long-term effects of invasive predatory lionfish (Pterois volitans) on Atlantic and Caribbean coral-reef communities. Env Biol Fishes 2011; DOI 10.1007/s10641-011-9795-1.

10. Green S, Akins J, Maljkovic M, Coté I. Invasive lionfish drive Atlantic coral reef fish declines. Plos One 2012; 7(3):e32596.
11. Luque G, Bellard C, Bertelsmeier C, Bonnaud E, Genovesi P, Simberloff D, Courchamp F. The $100^{\text {th }}$ of the world's worst invasive alien species. Biol Invasions 2013; 16(5):981985. DOI 10.1007/s10530-013-0561-5.

12. González J, Grijalba-Bendeck M, Acero P. A, Betancur-R R. The invasive red lionfish, Pterois volitans (Linnaeus 1758), in the southwestern Caribbean Sea. Aq Inv 2009; 4(3):507-510.

13. Mejía-Niño N, Garzón-Ferreira J. Dinámica de las interacciones alga-coral en dos bahías de la región de Santa Marta (Caribe colombiano) con distinto grado de influencia antropogénica. Bol Invest Mar Cost 2003; 32:243-261.

14. Díaz J, Barrios L, Cendales M, GarzónFerreira J, Geister J, López-Victoria M, et al. Áreas coralinas de Colombia. Invemar. Santa Marta, Serie Publicaciones Especiales 2000; 5:130-136.

15. Floeter SR, Krohling W, Gasparini JL. Ferreira CEL, Zalmon IR. Reef fish community structure on coastal islands of southeastern Brazil: the influence of exposure and benthic cover. Environ Biol Fish 2007; 78:147-160.

16. Luiz Jr OJ, Carvalho-Filho A, Ferreira CEL, Floeter SR, Gasparini JL, Sazima I. The reef fish assemblage of the Laje de Santos Marine State Park, Southwestern Atlantic: annotated checklist with comments on abundance, distribution, trophic structure, symbiotic associations, and conservation. Zootaxa 2008; 1807:1-25.

17. Krajewski JP, Floeter SR. Reef fish community structure of the Fernando de Noronha Archipielago (equatorial western Atlantic): the influence of exposure and benthic composition. Environ Biol Fish 2011; 92:25-40.

18. Grijalba-Bendeck M, Castañeda-Moya E, Acero P. A. Estructura de un ensamblaje íctico a fondos duros en el Caribe colombiano empleando la técnica del censo visual estacionario (CVE). Actual Biol 2004; 26(81):197-211.

19. Reyes-Nivia MC, Rodríguez-Ramírez A, Garzón-Ferreira J. Peces asociados a formaciones coralinas de cinco áreas del Caribe colombiano: Listado de especies y primeros registros para las áreas. Bol Invest Mar Cost 2004; 33:101-115. 
20. Westneat MW, Alfaro ME. Phylogenetic relationships and evolutionary history of the reef fish family Labridae. Mol Phylog Evol 2005; 36:370-390.

21. Olaya-Restrepo J, Reyes-Nivia MC, Rodríguez-Ramírez AR. Ensamblajes de peces arrecifales del área de Santa Marta y el Parque Nacional Natural Tayrona. Bol Invest Mar Cost 2008; 37(1):111-127.

22. Robertson DR. Interspecific competition controls abundance and habitat use of territorial Caribbean damselfishes. Ecology 1996; 77 885-899.

23. Maljkovic A, Van Leeuwen TE, Cove SN. Predation on the invasive red lionfish, Pterois volitans (Pisces: Scorpaenidae), by native groupers in the Bahamas. Coral Reefs 2008; 27:501.

24. Mumby, P.J, Harborne AR, Brumbaugh DR. Gruper as a natural biocontrol on invasive lionfish. 2011; PloS ONE 6(6):e21510. Doi: $10.1371 /$ journal. pone.0021510.

25. Lesser MP, Slattery M. Phase shift to algal dominated communities at mesophotic depths associated with lionfish (Pterois volitans) invasion on a Bahamian coral reef. Biol Invasions 2011; 13:1855-1868.
26. Morris JA, Akins JL. Feeding ecology of invasive lionfish (Pterois volitans) in the Bahamian archipelago. Environ Biol Fish 2009; 86:389-398.

27. Muñoz RC, Currin CA, Whitfield PE. Diet of invasive lionfish on hard bottom reefs of the southeast USA: insights from stomach contents and stable isotopes. Mar Ecol Prog Ser 2011; 432:181-193.

28. Muñoz-Escobar L, Gil-Agudelo D. Composición dietaría del pez león, Pterois volitans (Pisces: Scorpaenidae), en Santa Marta y el Parque Nacional Natural Tayrona. Bol Invest Mar Cost 2012; 41(2):471-477.

29. Whitfield PE, Hare JA, David AW, Harter SL, Muñoz RC, Addison M. Abundance estimates of the Indo-Pacific lionfish Pterois volitans/ miles complex in the western North Atlantic. Biol Invasions 2007; 9:53-64.

30. Munday PL, Jones GP, Caley MJ. Habitat specialization and the distribution and abundance of coral-dwelling gobies. Mar Ecol Prog Ser 1997; 152:227-239.

31. Gardiner M, Jones G. Habitat specialization and overlap in a guild of coral reef cardinalfishes (Apogonidae). Mar Ecol Prog Ser 2005; 305:163-175. 